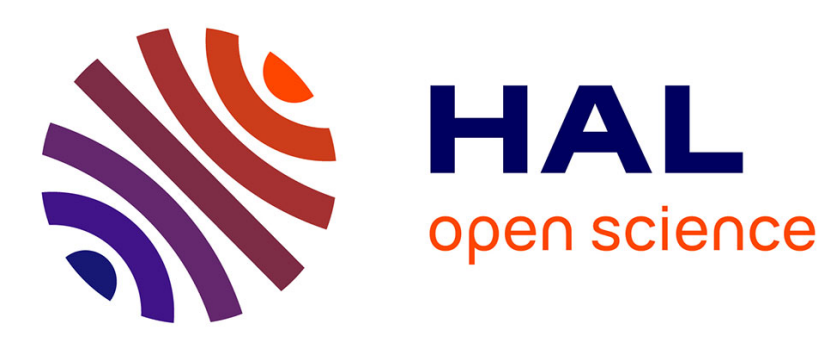

\title{
Optimal Location of Mobile Sensors for Environmental Monitoring \\ Didier Georges
}

\section{To cite this version:}

Didier Georges. Optimal Location of Mobile Sensors for Environmental Monitoring. ECC 2013 - 12th biannual European Control Conference, Jul 2013, Zurich, Switzerland. hal-00834642

\section{HAL Id: hal-00834642 \\ https://hal.science/hal-00834642}

Submitted on 17 Jun 2013

HAL is a multi-disciplinary open access archive for the deposit and dissemination of scientific research documents, whether they are published or not. The documents may come from teaching and research institutions in France or abroad, or from public or private research centers.
L'archive ouverte pluridisciplinaire HAL, est destinée au dépôt et à la diffusion de documents scientifiques de niveau recherche, publiés ou non, émanant des établissements d'enseignement et de recherche français ou étrangers, des laboratoires publics ou privés. 


\title{
Optimal Location of Mobile Sensors for Environmental Monitoring
}

\author{
Didier Georges
}

\begin{abstract}
This paper is devoted to the optimal location of a mobile sensor network for environmental monitoring applications. Effective pollution monitoring in the atmosphere or in hydraulic systems (rivers, lakes or seas) with a limited number of sensors is a very challenging issue. In this paper, we will only consider physical phenomena governed by the so-called advection-diffusion partial-differential equation (ADPDE). The paper goal is to derive a simple potential-based navigation strategy ensuring the optimal location of a mobile sensor network. The optimal location of the sensors is determined through the maximization of an observability index based on the observability gramian of the ADPDE derived from a spectral method. The application to the control of a 20 -sensor network applied to a $2 \mathrm{D}$ air pollution model demonstrates the effectiveness of the proposed approach. We can also emphasize the fact that the here-proposed methodology can be easily applied to the optimal location of static sensors.
\end{abstract}

\section{INTRODUCTION}

Sensors networks are now well recognized for their potential ability to monitor or track distributed environmental phenomena (weather, seismic events, wildfires, air, soil or river pollution ...) Presently, monitoring systems are mostly based on static networks of sensors which have to be optimally located [11]. A natural extension of sensor networks is the use of mobile sensors, which can potentially provide more versatility in collecting distributed information. An additional advantage of mobile sensor networks is their exploration capability in hazardous environments.

Most of the existing approaches used in environmental monitoring exploit ad hoc systematic exploration techniques by using concentration gradient and flow direction to track plums to their source [2], [15], [19]. However in the infinitedimensional framework, we can mention research works [3], [4], [5] whose main goal is to derive a stable distributed parameter state observer by using measurements from some mobile sensors which have also to be controlled to satisfy this goal. Rather that using statistical field reconstruction methods (see [12] for instance), the use of an observability index based on the so-called observability gramian of a reduced model of the PDE is proposed. The objective is mainly to get an optimal configuration of the sensors suitable to enhance the performance of state observers designed for estimation or prediction of the distributed pollution dynamics, including the derivation of the pollution source location. Then a navigation strategy of a mobile sensor network is proposed which maximizes the observability index while providing a optimal configuration of the sensors

D. Georges is with GIPSA-lab, Grenoble Institute of Technology, UJF, U. Stendhal, CNRS, France, didier.georges at grenoble-inp.fr under sensor interdistance constraints. However we will not discuss the issue of designing appropriate state observers for environmental monitoring capable of taking advantage of the optimal sensor location procedure. The field of potential applications of the here-proposed approach is very large and is not limited to the case of advection-diffusion PDEs : Monitoring of pollution advection-diffusion in 1D or 2D hydraulic systems coupled to Saint-Venant equations (rivers, lakes, estuaries or seas), underseas pollution monitoring, 2D or 3D air pollution monitoring, seismic monitoring, or monitoring of large infrastructures in civil engineering (dams for instance), for some important examples. Reference [16] provides a deep insight on environmental monotoring of such systems.

The organization of the paper is now as follows. In section II, we introduce the advection-diffusion problem that we will consider, together with a spectral method leading to a reduced model in finite dimensions. Section III is devoted to the derivation of the optimal location problem based on the notion of observability gramian. The navigation strategy of a team of mobile sensors is then presented in section IV. Simulations results are presented in section $\mathrm{V}$ followed by some conclusions in section VI.

\section{POLLUTION MODELING}

\section{A. The advection-diffusion PDE}

Pollution dispersion (without chemical reaction) may be well modeled (see [14] and [18] for instance, in the case of air pollution) on a domain $\Omega$ by an advection-diffusion partial differential equation of the form:

$$
\frac{\partial z}{\partial t}(x, t)+U(x, t) \cdot \nabla z(x, t)=K \triangle z(x, t)+D(x, t) S(t)
$$

where $x \in \Omega \subset \mathbb{R}^{N}$, with $N=1,2$ or $3, z(x, t)$ is the concentration of a chemical species (the pollutant), $U(x, t)$ is a vector of flow velocities which is supposed to be known (through measurements or computation of other PDEs, such as the Saint-Venant equations governing the dynamics of open-channel hydraulic systems or meteorological models in the case of air pollution), $K$ is a constant diffusion coefficient which is supposed to be known, and $D(x, t)$ is the operator defining how the source of pollutant $S(t)$ acts in the domain $\Omega$. $\nabla$ and $\triangle$ stand for the gradient and the Laplacian respectively. "." denotes the standard scalar product in $\mathbb{R}^{2}$.

Some initial conditions $z(x, t=0)=z_{0}(x)$ and some boundary conditions on the domain boundary $\partial \Omega$ have to be provided. In this paper and without restriction, some Dirichlet boundary conditions will be considered:

$$
z(x, t)=z_{b c}(x, t), \forall x \in \partial \Omega \text {. }
$$




\section{B. A reduced-order model based on a spectral method}

Rather than using a standard finite-difference method based on some spatial-grid dependent approximate solutions, which will lead to differentiability issues in the derivation of the mobile sensor control strategy, a spectral method (known for providing accurate solutions [7]) is proposed with the goal of deriving a reduced-order finite-dimensional model of the ADPDE, which does not rely on a spatial grid.

It consists in seeking an approximate $z_{a}(x, t)$ of (1)-(2) as a linear combination of $N$ basis functions $\phi_{i}(x), i=1, \ldots, N$ in a $N$-dimensional subspace:

$$
z_{a}(x, t)=\sum_{i=1}^{N} z_{i}(t) \phi_{i}(x),
$$

where the $z_{i}(t)$ 's are the coordinates in the function basis.

The problem consists now in introducing (3) in both (1) and (2) to derive two residuals:

$$
\begin{aligned}
R(x, t) & =\frac{\partial z_{a}}{\partial t}(x, t)+U(x, t) \cdot \nabla z_{a}(x, t) \\
& -K \triangle z_{a}(x, t)-D(x, t) S(t), \forall x \in \Omega, \\
\bar{R}(x, t) & =z_{a}(x, t)-z_{b c}(x, t), \forall x \in \partial \Omega .
\end{aligned}
$$

The problem is now to render these residuals smaller as possible (ideally equal to zero). In order to get this result, a solution will consist in computing $z_{a}(x, t)$ such that both $R$ and $\bar{R}$ are orthogonal to each basis function $\phi_{i}(x)$ :

$$
\begin{aligned}
\int_{\Omega} R(x, t) \phi_{j}(x) d \Omega & =0, j=1, \ldots, N \\
\int_{\partial \Omega} \bar{R}(x, t) \phi_{j}(x) d \partial \Omega & =0 .
\end{aligned}
$$

These orthogonality conditions can be also simplified as:

$$
\begin{array}{r}
\int_{\Omega} R(x, t) \phi_{j}(x) d \Omega+\gamma \int_{\partial \Omega} \bar{R}(x, t) \phi_{j}(x) d \partial \Omega=0, \\
j=1, \ldots, N,
\end{array}
$$

where $\gamma$ is any real coefficient non equal to zero.

By introducing the expression of each residual, we get

$$
\begin{array}{r}
\int_{\Omega}\left[\frac{\partial z_{a}}{\partial t}(x, t)+U(x, t) . \nabla z_{a}(x, t)-K \triangle z_{a}(x, t)\right. \\
-D(x, t) S(t)] \phi_{j}(x) d \Omega \\
+\gamma \int_{\partial \Omega}\left[z_{a}(x, t)-z_{b c}(x, t)\right] \phi_{j}(x) d \partial \Omega=0, \\
j=1, \ldots, N .
\end{array}
$$

Since all the operators are linear, the orthogonality conditions (9) leads to the solution of a linear dynamical system expressed in the coordinates $z_{a}(t)=\left(z_{a}^{1}(t), \ldots, z_{a}^{N}(t)\right)^{T}$ :

$$
E \dot{z}_{a}(t)=A(t) z_{a}(t)+B(t) S(t)+F(t), z_{a}(0)=z_{a}^{0},
$$

where the matrices $E, A$ and the vectors $B$ and $F$ have elements defined as follows:

$$
E_{i j}=\int_{\Omega} \phi_{i}(x) \phi_{j}(x) d \Omega
$$

$$
\begin{array}{r}
A_{i j}(t)=\int_{\Omega}\left[-U(x, t) . \nabla \phi_{i}(x)+K \triangle \phi_{i}(x)\right] \phi_{j}(x) d \Omega \\
+\gamma \int_{\partial \Omega} \phi_{i}(x) \phi_{j}(x) d \partial \Omega \\
B_{j}(t)=\int_{\Omega} D(x, t) \phi_{j}(x) d \Omega
\end{array}
$$

and

$$
F_{j}(t)=-\gamma \int_{\partial \Omega} z_{b c}(x, t) \phi_{j}(x) d \partial \Omega
$$

and where the initial state vector $z_{a}^{0}$ is computed as the solution of the linear system

$$
\begin{array}{r}
\int_{\Omega}\left[z_{a}(x, 0)-z_{0}(x)\right] \phi_{j}(x) d \Omega \\
+\gamma \int_{\partial \Omega}\left[z_{a}(x, 0)-z_{b c}(x, 0)\right] \phi_{j}(x) d \partial \Omega=0, \\
j=1, \ldots, N .
\end{array}
$$

which is equivalent to

$$
G z_{a}^{0}+H=0,
$$

where $G$ and $H$ are defined by

$$
\begin{aligned}
& G_{i j}=\int_{\Omega} \phi_{i}(x) \phi_{j}(x) d \Omega \\
& +\gamma \int_{\partial \Omega} \phi_{i}(x) \phi_{j}(x) d \partial \Omega
\end{aligned}
$$

and

$$
\begin{array}{r}
H_{j}=-\int_{\Omega} z_{0}(x) \phi_{j}(x) d \Omega \\
-\gamma \int_{\partial \Omega} z_{b c}(x, 0) \phi_{j}(x) d \partial \Omega .
\end{array}
$$

This approach is very well known as the Garlerkin weightedresidual method.

If the basis functions $\phi_{i}(x)$ 's are chosen to be orthogonal on the domain $\Omega$ :

$$
E_{i j}=\int_{\Omega} \phi_{i}(x) \phi_{j}(x) d \Omega=0, \forall i, j, i \neq j
$$

then $E$ is a diagonal matrix which can be easily inverted. It also follows that the problem is better numerically conditioned.

For example, in the case of 1D problems defined on a domain $\Omega=[-1,+1]$, Legendre's polynomials given by the sequence $\left\{L_{i}(x)\right\}_{i \geq 0}$

$$
\begin{array}{r}
(i+1) L_{i+1}(x)=(2 i+1) x L_{i}(x)-i L_{i-1}(x), \\
L_{0}(x)=1, L_{1}(x)=x,
\end{array}
$$

which are orthogonal on $[-1,+1]$ with unitary weight:

$$
\int_{-1}^{1} L_{i}(x) L_{j}(x) d x=0, \forall i, j, i \neq j
$$

and

$$
\int_{-1}^{1} L_{i}(x) L_{i}(x) d x=\frac{2}{2 i+1}, \forall i \geq 0,
$$

can be used instead of the conventional polynomial approximants $\phi_{i}(x)=x^{i}$. 
For multidimensional problems, the basis functions can be defined as products in each dimension. For instance, in the 2D case (using spatial coordinates $(x, y)$ ), we get

$$
\begin{array}{r}
\phi_{k}(x, y)=L_{i}(x) \times L_{j}(y), k=i+(j-1) N, \\
i, j=1, \ldots, N .
\end{array}
$$

This is precisely the choice of basis functions made for the air pollution application presented in this paper.

\section{Some remarks.}

- Of course, many other basis functions can be used, such as wavelets, which are known to exhibit very nice multiresolution properties.

- $\gamma$ has to be chosen in order to ensure that $A$ is Hurwitz. If the basis functions are chosen such that the boundary conditions (2) are satisfied, $\gamma$ is equal to zero.

- It can be interesting to introduce a weak formulation of the Laplacian operator (using Green's formula):

$$
\int_{\Omega} \triangle u \cdot v d \Omega=-\int_{\Omega} \nabla u \cdot \nabla v d \Omega+\int_{\partial \Omega} \nabla u \cdot v n_{x} d \partial \Omega
$$

in order to reduce the continuity conditions on the basis functions. In this case, the $A_{i j}$ 's become

$$
\begin{aligned}
A_{i j}(t)= & \int_{\Omega}\left[-U(x, t) \cdot \nabla \phi_{i}(x) \phi_{j}(x)\right. \\
& \left.-K \nabla \phi_{i}(x) \cdot \nabla \phi_{j}(x)\right] d \Omega \\
+ & \int_{\partial \Omega} \nabla \phi_{i}(x) \cdot \phi_{j}(x) n_{x} d \partial \Omega \\
+ & \gamma \int_{\partial \Omega} \phi_{i}(x) \phi_{j}(x) d \partial \Omega
\end{aligned}
$$

- The infinite-dimensional problem (1)-(2) is known to be exponentially stable.

\section{AN OBSERVABILITY-BASED POTENTIAL FUNCTIONAL FOR MOBILE SENSOR NAVIGATION}

\section{A. Some background on observability function and gramian}

We start this section with some background on observability function [1].

We consider some continuous-time time-varying linear systems described in state-space form by

$$
\begin{aligned}
& \dot{x}=A(t) x \\
& y=C(t) x
\end{aligned}
$$

where $x \in R^{n}$ and $y \in R^{p}$. We assume that the system is at least detectable.

The so-called "transient observability function" is defined as

$$
L_{o}(X, T)=\frac{1}{2} \int_{0}^{T}\|y(t)\|^{2} d t, x(0)=X,
$$

that is the output energy generated by any initial state $X \in R^{n}$ in the time interval $[0, T] . L_{o}$ may be rewritten as

$$
L_{o}(X, T)=\frac{1}{2} X^{T}\left\{\int_{0}^{T} \Phi^{T}(t, 0) C(t)^{T} C(t) \Phi(t, 0) d t\right\} X
$$

with $\frac{d \Phi(t, \tau)}{d t}=A(t) \Phi(t, \tau), \Phi(t, t)=I_{d}$.

A necessary and sufficient condition for observability (resp. detectability) of the pair $(C(t), A(t))$ is that there exists $\forall t \in[0, T]$, a positive definite (resp. non negative definite) symmetric matrix $W(t)$, solution to the following differential Lyapunov equation:

$$
\begin{aligned}
-\dot{W}(t)+A^{T}(t) W(t)+W(t) A(t) & =-C^{T}(t) C(t), \\
W(0) & =0,
\end{aligned}
$$

so-called "observability gramian" matrix, such that

$$
L_{o}(X, T)=\frac{1}{2} X^{T} W(T) X .
$$

In the case of time-invariant asymptotically stable observable (resp. detectable) linear systems defined by the pair $(C, A), L_{o}$ is finite when $T \rightarrow+\infty$ and $\lim _{T \rightarrow+\infty} W(T)=\bar{W}$. $\bar{W}$ is obtained as the unique positive definite (resp. non negative definite) solution to the Lyapunov problem:

$$
A^{T} W+W A=-C^{T} C .
$$

It follows that $W(T)$ or $\bar{W}$ can be used as a measure of the observability degree of the system, since the singular values of $W$ or $\bar{W}$ represent the sensitivity of output $y$ to each component of any initial state $x(0)=X$.

Since our goal is to maximize the observability of a physical phenomenon, we seek for maximizing a norm of the observability gramian [9]; for instance, the trace of the gramian that is the sum of the singular values of the gramian:

$$
I_{o}(0, T)=\operatorname{trace}(W(T)),
$$

where $I_{o}$ will be denoted as the transient observability index, while $I_{a o}=I_{o}(0,+\infty)=\operatorname{trace}(\bar{W})$ will be denoted as the asymptotic observability index in what follows.

B. An observability index for the reduced model of the $A D P D E$

The previously-described approach can be easily applied to reduced model (10), where output $y$ corresponds to the measurement vector of the pollutant $z(x, t)$ provided by a set of $\mathrm{M}$ mobile sensors:

$$
y(t)=\left(z\left(x_{1}(t), t\right), \ldots, z\left(x_{M}(t), t\right)\right)^{T},
$$

where $x_{i}(t)$ is the coordinate vector of each mobile sensor $i$, $i=1, \ldots, M$. in domain $\Omega$, at time $t$.

$$
z\left(x_{i}, t\right)=\int_{\Omega} \delta\left(x-x_{i}\right) z(x, t) d x,
$$

where $\delta$ denotes the spatial Dirac-delta function.

In our approach, $z$ is approximated by $z_{a}(x, t)=$ $\sum_{\substack{i=0 \\ \text { by }}}^{N} z_{i}(t) \phi_{i}(x)$. Therefore, the $(M \times N)$ output matrix is given

$$
C(t)=\left(\begin{array}{ccccc}
\phi_{1}\left(x_{1}(t)\right) & \cdot & \cdot & \cdot & \phi_{N}\left(x_{1}(t)\right) \\
\cdot & & & & \cdot \\
\cdot & & & & \cdot \\
\cdot & & & & \cdot \\
\phi_{1}\left(x_{M}(t)\right) & \cdot & \cdot & \cdot & \phi_{N}\left(x_{M}(t)\right)
\end{array}\right)
$$


Now we use the following assumption in what follows:

Assumption: the time-varying velocity field $U(x, t)$ is replaced by a mean velocity field defined over a finite time interval $[0, T]$

$$
U(x)=\frac{1}{T} \int_{0}^{T} U(x, t) d t .
$$

This assumption means that the velocity field $U(x, t)$ is available through measurements or computation over $[0, T]$.

By using this assumption, matrix $A$ of (10) is no more time-varying and the observability index $I_{a o}$ can be computed by considering any fixed configuration $\left(x_{1}, \ldots, x_{M}\right)$ of the $M$ sensors in domain $\Omega . \bar{W}\left(x_{1}, \ldots, x_{M}\right)$ is computed as solution to the following Lyapunov equation:

$$
H^{T} W+W H=-C\left(x_{1}, \ldots, x_{M}\right)^{T} C\left(x_{1}, \ldots, x_{M}\right),
$$

where $H=E^{-1} A$ is an Hurwitz matrix and $I_{a o}\left(x_{1}, \ldots, x_{M}\right)=$ $\operatorname{trace}\left(\bar{W}\left(x_{1}, \ldots, x_{M}\right)\right)$.

Remark. The observability index does neither depend on the magnitude of the source nor its location $D(x, t)$ in domain $\Omega$, represented by vector $B$.

\section{Sensor interdistance constraints}

Two kinds of interdistance constraints should be introduced: On the one hand, some minimum distance between each sensor has to be considered since the sensors should not be located at the same place. On the other hand, some communication constraints between the sensors should be taken into account, since communications between sensors will be needed in our case to compute the trajectory of each mobile sensor.

These sensor interdistance constraints will be taken into account by using a barrier function approach [6], which is very useful in constrained optimization as a penalty functional. If $d_{i j}$ is the distance between two mobile sensors $i$ and $j$, we can define the following potential $F_{d}$ :

$$
F_{d}=-\mu \sum_{i=1}^{M-1} \sum_{j=i+1}^{M}\left\{\log \left(d_{i j}^{2}-\alpha^{2}\right)+\log \left(\beta^{2}-d_{i j}^{2}\right)\right\},
$$

where $\mu>0, \alpha$ and $\beta$ are the minimum and maximum distances between two robots respectively, and $d_{i j}=\left\|x_{i}-x_{j}\right\|$ ( $\|$.$\| is the Euclidian norm).$

This potential ensures that the mobile sensor configuration is always such that $\alpha<d_{i j}<\beta, \forall i, j=1, \ldots, M$.

\section{Optimal static configuration of the mobile sensors}

The optimal static configuration of the M-mobile sensor network is obtained as solution to the following optimization problem:

$$
\min _{x_{i}, i=1, \ldots, M}-I_{a o}\left(x_{1}, \ldots, x_{M}\right)+F_{d}\left(x_{1}, \ldots, x_{M}\right),
$$

that is

$$
\begin{array}{r}
\min _{x_{i}, i=1, \ldots, M}-\operatorname{trace}\left(\bar{W}\left(x_{1}, \ldots, x_{M}\right)\right) \\
-\mu \sum_{i=1}^{M-1} \sum_{j=i+1}^{M}\left\{\log \left(d_{i j}^{2}-\alpha^{2}\right)+\log \left(\beta^{2}-d_{i j}^{2}\right)\right\} .
\end{array}
$$

This problem may be viewed as a multi-criteria optimization problem whose solution $x_{i}^{*}, i=1, \ldots, M$ is a trade-off between observability maximization and generation of an equilibrium configuration corresponding to fixed sensor interdistances defined in interval $[\alpha, \beta] . \mu$ is used to tune this trade-off.

Proposition: Any equilibrium configuration is necessarily solution of the algebraic set of nonlinear equations:

$$
\begin{array}{r}
-\nabla_{x_{i}} I_{a o}\left(x_{1}, \ldots, x_{M}\right) \\
-2 \mu \sum_{j=1, j \neq i}^{M}\left\{\frac{\left(x_{i}-x_{j}\right)}{d_{i j}^{2}-\alpha^{2}}-\frac{\left(x_{i}-x_{j}\right)}{\beta^{2}-d_{i j}^{2}}\right\}=0, \\
i=1, \ldots, M-1 .
\end{array}
$$

Proof. The set of equations (41) corresponds to Lagrange necessary conditions for optimality of problem (40).

Remark. From the barrier function theory [6], it appears that, for all $\mu$ sufficiently small, problem (40) admits a minimizer near a solution to the constrained optimization problem:

$$
\begin{array}{r}
\min _{x_{i}, i=1, \ldots, M}-\operatorname{trace}\left(\bar{W}\left(x_{1}, \ldots, x_{M}\right)\right) \\
\text { s.t. } \alpha \leq d_{i j} \leq \beta, \forall i=1, \ldots, M-1, j=i+1, \ldots, M
\end{array}
$$

\section{THE NAVIGATION STRATEGY}

In this paper, the dynamics of the mobile sensors will not be considered and the problem will be the real-time planning of the trajectory of each mobile sensor by using a potentialbased approach [13], [8], which is well known to be easily implementable.

\section{A. A potential-based control strategy}

In this approach, the trajectory $\left\{x_{i}^{k}\right\}_{k \geq 0}$ inside the domain $\Omega$ of each sensor $i, i=1, \ldots, M$ is generated by a gradient method:

$$
x_{i}^{k+1}=x_{i}^{k}-\rho \nabla_{x_{i}} P\left(x_{1}^{k}, \ldots, x_{i-1}^{k}, x_{i}^{k}, x_{i+1}^{k}, \ldots, x_{M}^{k}\right),
$$

where $P$ is a given potential functional, $\rho$ is the gradient step which has to be chosen in order that $P\left(x_{1}^{k+1}, \ldots, x_{i-1}^{k+1}, x_{i}^{k+1}, x_{i+1}^{k+1}, \ldots, x_{M}^{k+1}\right)<$ $P\left(x_{1}^{k}, \ldots, x_{i-1}^{k}, x_{i}^{k}, x_{i+1}^{k}, \ldots, x_{M}^{k}\right)$.

The common goal of all the sensors is therefore to reach an optimal configuration $x_{i}^{*}, i=1, \ldots, M$ such that the gradient of the potential $P$ is equal to zero, that is a solution to the necessary conditions of the optimization problem

$$
\min _{x_{i}, i=1, \ldots, M} P\left(x_{1}, \ldots, x_{M}\right) \text {. }
$$

Then in our case, the choice of the functional $P$ is naturally given by

$$
P\left(x_{1}, \ldots, x_{M}\right)=-I_{o}\left(x_{1}, \ldots, x_{M}\right)+F_{d}\left(x_{1}, \ldots, x_{M}\right) .
$$

Finally the trajectory $x_{i}^{k}$ of each sensor $i$ is obtained from the following recursive equation

$$
\begin{aligned}
x_{i}^{k+1}= & x_{i}^{k}+\rho\left[\nabla_{x_{i}} I_{a o}\left(x_{1}^{k}, \ldots, x_{M}^{k}\right)\right. \\
& \left.+2 \mu \sum_{j=1, j \neq i}^{M}\left\{\frac{\left(x_{i}^{k}-x_{j}^{k}\right)}{d_{i j}^{2}-\alpha^{2}}-\frac{\left(x_{i}^{k}-x_{j}^{k}\right)}{\beta^{2}-d_{i j}^{2}}\right\}\right] .
\end{aligned}
$$


The navigation will end when $\left\|x^{k+1}-x^{k}\right\|<\varepsilon_{s}$, where $\varepsilon_{s}$ is a given reached precision.

It can be easily checked that any cluster point of the sequence $x_{i}^{k}, i=1, \ldots, M$ (a final configuration reached) corresponds to a solution satisfying necessary conditions (41).

\section{Some remarks.}

- if the basis functions are regular, output matrix $C$ is differentiable and $\nabla_{x_{i}} I_{a o}\left(x_{1}, \ldots, x_{M}\right)$ is always well defined as

$$
\nabla_{x_{i}} I_{a o}\left(x_{1}, \ldots, x_{M}\right)=\operatorname{trace}\left(W_{i}^{\prime}\right),
$$

where $W_{i}^{\prime}$ is solution to the Lyapunov equation

$$
H^{T} W_{i}^{\prime}+W_{i}^{\prime} H=-2 \frac{\partial C_{i}{ }^{T}}{\partial x_{i}} C_{i},
$$

where $C_{i}$ is the ith row of matrix $C$.

- Each mobile sensor $i, i=1, \ldots, M$ computes its own component of the gradient vector of the potential functional at step $k$. However the current positions of the $M-1$ other sensors at step $k$ have to be transmitted and received by sensor $i$ to compute this gradient component. This is the only information needed. It should be also assumed that an ad hoc communication infrastructure is implemented in the sensor network.

- With this navigation strategy (mainly by tuning the gradient step $\rho$ ), we will be able to track slow variations of observability index $I_{a o}$ induced by (slow w.r.t. sensor kinematics) variations of the mean velocity field, by updating the position of each sensor according to (47).

\section{Algorithm for slowly time-varying velocity field track-} ing:

$$
\begin{aligned}
x_{i}^{k+1}= & x_{i}^{k}+\rho\left[-\nabla_{x_{i}} \operatorname{trace}\left(W\left(t_{k}\right)\right)\right. \\
& \left.+2 \mu \sum_{j=1, j \neq i}^{M}\left\{\frac{\left(x_{i}^{k}-x_{j}^{k}\right)}{d_{i j}^{2}-\alpha^{2}}-\frac{\left(x_{i}^{k}-x_{j}^{k}\right)}{\beta^{2}-d_{i j}^{2}}\right\}\right] . \\
\frac{\partial}{\partial t} Z(x, t)= & L(Z(x, t))+f(t) \\
B(Z(x, t))= & 0, Z(x, 0)=Z_{0}(x) \\
\dot{W}(t)= & A^{T}(t) W(t)+W(t) A(t) \\
& +C^{T}\left(x_{1}^{k}, \ldots, x_{M}^{k}\right) C\left(x_{1}^{k}, \ldots, x_{M}^{k}\right) \\
W(0)= & 0
\end{aligned}
$$

where $L$ denotes a differential operator, $B$ is a boundary condition operator and $f$ is a bounded operator. $(L, f, B)$ defines the PDEs governing $Z(x, t)$ which includes the velocity field $U(x, t)$ over the domain (for instance the shallow water equations in the case of water pollution, with $Z(x, t)=$ $(h(x, t), U(x, t))$, where $h$ is the water level and $U$, the water velocity). It should be also notice that a differential observability gramian Lyapunov equation is needed since the PDE is time-varying (reduced-model state matrix $A$ is now time-varying, since the velocity field $U$ is). $t_{k}$ is the time instant corresponding to navigation iteration $k$.

\section{B. Discussion on an improved navigation strategy}

In order to relax the assumption of constant or slowly timevarying velocity field, we can derive an improved navigation strategy, solution to the following optimal control problem defined on a given time interval $[0, T]$ :

$$
\begin{array}{r}
\min _{u_{i}, i=1, \ldots, M} \int_{0}^{T}\left[-\operatorname{trace}\left(W\left(t_{k}\right)\right)+\sum_{i=1}^{M} u_{i}^{T}(t) R_{i} u_{i}(t)\right. \\
\left.-\mu \sum_{i=1}^{M-1} \sum_{j=i+1}^{M}\left\{\log \left(d_{i j}^{2}(t)-\alpha^{2}\right)+\log \left(\beta^{2}-d_{i j}^{2}(t)\right)\right\}\right] d t \\
-\mu \sum_{i=1}^{M-1} \sum_{j=i+1}^{M}\left\{\log \left(d_{i j}^{2}(T)-\alpha^{2}\right)+\log \left(\beta^{2}-d_{i j}^{2}(T)\right)\right\} \\
-\operatorname{trace}(W(T)) \\
s . t . \ddot{x}_{i}(t)=u_{i}(t), i=1, \ldots, M, \\
\dot{x}_{i}(T)=0, \\
-W(t)+A^{T}(t) W(t)+W(t) A(t) \\
=-C^{T}\left(x_{1}(t), \ldots, x_{M}(t)\right) C\left(x_{1}(t), \ldots, x_{M}(t)\right), \\
W(0)=0, \forall t \in[0, T],
\end{array}
$$

where $u_{i}$ is the acceleration vector of mobile sensor $i$ and $R_{i}$ is the weighting matrix of accelerations. ${ }^{1}$.

This advanced strategy will not be experimented in this paper.

\section{APPLICATION TO 2D AIR POLLUTION}

Now we consider the optimal navigation of a M-sensor network towards the equilibrium configuration satisfying necessary conditions (41), on domain $\Omega=[-1 \mathrm{~km}, 1 \mathrm{~km}]^{2}$. The ADPDE parameters used in the simulation are given in table I, where the velocity field $U=\left(U_{x}, U_{y}\right)$ is supposed to be uniform over the domain and constant with respect to time.

TABLE I

ADPDE PARAMETERS

\begin{tabular}{|c||c||c||c||c|}
\hline$U_{x}$ & $U_{y}$ & $K$ & B.C. & $D(x, t)$ \\
\hline $10 \mathrm{~km} / \mathrm{h}$ & $10 \mathrm{~km} / \mathrm{h}$ & 1 & $z_{b c}(x, t)=0$ & $e^{-\frac{\|x\|^{2}}{2 \sigma^{2}}}, \sigma=0.01$ \\
\hline
\end{tabular}

The reduced model of the ADPDE is obtained by using 20 Legendre polynomials in each spatial direction as basis functions and with $\gamma=-1 e^{4}$.

In the simulation depicted in Fig. 1, the initial positions of the sensors are randomly distributed over the domain. The gradient-based navigation approach (47) is applied to reach an optimal configuration denoted by some small circles, with the parameters given in table II. Sensor interdistance constraints are satisfied thanks to the potential $F_{d}$.

Fig. 2 presents how well the sensor network adapts itself to a change in the wind velocity $\left(U_{x}, U_{y}\right)$ that changes from $(10 \mathrm{~km} / \mathrm{h}, 10 \mathrm{~km} / \mathrm{h})$ to $(-10 \mathrm{~km} / \mathrm{h}, 5 \mathrm{~km} / \mathrm{h})$. The initial configuration of the sensors corresponds to the final configuration in Fig. 1.

\footnotetext{
${ }^{1}$ Rather than using a double integrator dynamics, we could introduce a more complex dynamical model of each mobile sensor without restriction.
} 
TABLE II

PARAMETERS OF THE POTENTIAL NAVIGATION

\begin{tabular}{|c||c||c||c||c|}
\hline$\rho$ & $\alpha$ & $\beta$ & $\varepsilon_{s}$ & $\mu$ \\
\hline 0.2 & $0.01 \mathrm{~km}$ & $3 \mathrm{~km}$ & $1 e^{-3}$ & 0.001 \\
\hline
\end{tabular}

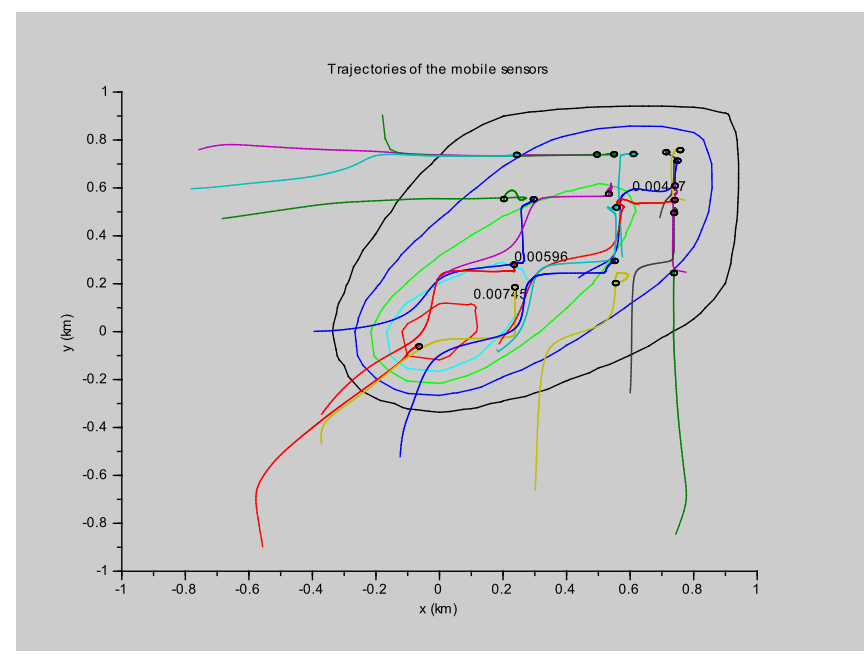

Fig. 1. Trajectories of the 20-sensor network with level curves corresponding to the equilibrium ADPDE solution (reduced-model field).

It appears that the sensors tend to localise themselves at extremities of the concentration field.

\section{CONCLUSIONS}

In this paper, a novel approach has been proposed for the goal of real-time planning of a mobile sensor network for environmental monitoring applications in either $1 \mathrm{D}, 2 \mathrm{D}$ or 3D. The control goal is to reach an optimal location of the sensors in order to maximize an observability gramian-based index under sensor interdistance constraints. Application to a simple 2D example with a team of 20 mobile sensors has demonstrated the effectiveness of this approach. The here-

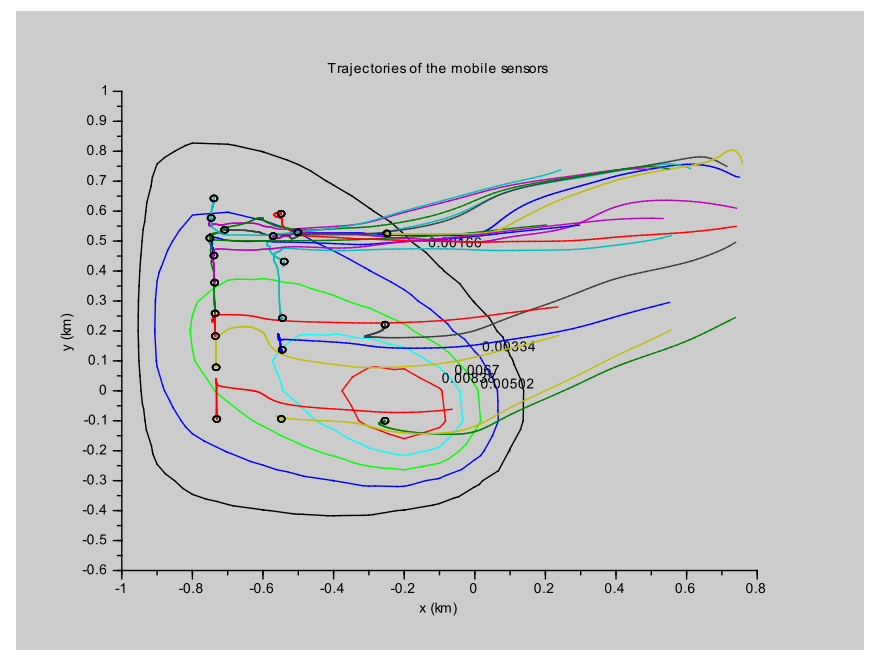

Fig. 2. Adaptation of 20-sensor network to a change of the wind velocity. proposed methodology can be also applied to the problem of optimally locating static sensors. Further researches will be devoted to including energy and/or communication constraints, as an extension of the work in [10], but also to inclusion of robustness w.r.t. measurement noise. Another research perspective is the derivation, the effective computation and the use of infinite-dimensional observability gramian, as in [17] for the linear advection PDE.

\section{REFERENCES}

[1] Brockett, R.W., Finite dimensional linear systems. New York: Wiley, 1970.

[2] Cortes, J., Martinez, S., Karatas, T., and Bullo, F., Coverage control for mobile sensing networks. IEEE Transactions on Robotics and Automation, Volume: 20 Issue: 2, April 2004.

[3] Demetriou, M.A., Guidance of mobile actuator-plus-sensor networks for improved control and estimation of distributed parameter systems. IEEE Trans. on Automatic Control, Vol. 55, 7, 1570-1584, 2010.

[4] Demetriou, M.A., and Ucinski, D., State estimation of spatially distributed processes using mobile sensing agents. Proceedings of the American Control Conference, 2011.

[5] Demetriou, M.A., Gatsonis, N.A., and Court, J., Numerical studies of concentration estimation and source tracking of a gaseous source using multiple mobile distributed sensors. Proceedings of the 20th Mediterranean Conference on Control and Automation, 2012.

[6] Fiacco, A.V., and McCormick, G.P., Nonlinear programming: Sequential unconstrained minimization techniques. SIAM Publications, 1990.

[7] Fletcher C.A.J., Computational Galerkin methods, Springer Series in Computational Physics. Springer-Verlag, 1984.

[8] Gazi, V., and Fidan, B., Coordination and control of multi-agent dynamic systems: Models and approaches. Lecture notes in Computer Science, Springer, 2007.

[9] Georges, D., The use of observability and controllability gramians or functions for optimal sensor and actuator location in finite-dimensional systems. Proceedings of the 34th IEEE Conference on Decision and Control, 1995.

[10] Georges, D., Energy minimization and observability maximization in multi-hop wirelesss sensor networks. Proceedings of the IFAC Word Congress, Milano, Italy, 2011.

[11] Ghanem, M., Guo Y., Hassard, J., Osmond, M., Richards, M., Sensor Grids For Air Pollution Monitoring. In Proc. 3rd UK e-Science All Hands Meeting, 2004.

[12] Gneiting T., Nonseparable, stationary covariance functions for spacetime data. J. Am. Statist. Assoc. 97, 590-600, 2002.

[13] Kowalczyk, W., and Koslovski, K., Artificial potential based control for a large-scale formation of robots. Climbing and walking robots, Part. II, pp. 191-199, Springer, 2005.

[14] Tirabassi, T., Analytical air pollution advection and diffusion models. Water, Air, \& Soil Pollution, Volume 47, Numbers 1-2, september 1989.

[15] Trincavelli, M., Reggente, M.,Coradeschi, S., Loutfi, A., Ishida, H., Lilienthal A.J., Towards environmental Monitoring with mobile robots. Proceedings of the IEEE/RSJ International Conference on Intelligent Robots and Systems, 2008.

[16] Ucinski, D., Optimal Measurement Methods for Distributed Parameter System Identification. CRC Press, Boca Raton, 2005, ISBN: 0-84932313-4.

[17] Vaidya, U., Rajarm, R., and Dasgupta, S., Actuator and sensor placement in linear advection PDE. Proceedings of the 50th CDCECC, Orlando, USA, 2011.

[18] Zannetti, P., Air Pollution Modelling. Theories, Computational Methods and Available Software. Computational Mechanics Publications, Southampton, Boston, Van Nostrand Reinhold, New York, 1990.

[19] Zarzhitsky, D., Spears, D. F., and Spears W., Distributed Robotics Approach to Chemical Plume Tracing. Proceedings of the IEEE/RSJ International Conference on Intelligent Robots and Systems, 2005. 